\title{
Baby Buddy app evaluation-effective uplift in breast feeding despite unclear mechanism
}

\author{
Mitch Blair ${ }^{1}$, Soo Downe ${ }^{2}$, Adrienne Gordon ${ }^{3}$, Loretta Musgrave ${ }^{4}$, Caroline Homer ${ }^{5}$, Claire Etherington ${ }^{6}$ \\ ${ }^{1}$ Department of Primary Care and Public Health, Imperial College, London, UK; ${ }^{2}$ Research in Childbirth and Health (ReaCH) Group, University \\ of Central Lancashire, Preston, UK; ${ }^{3}$ Department of Obstetrics, Gynaecology and Neonatology, Royal Prince Alfred Hospital, Camperdown, NSW, \\ Australia; ${ }^{4}$ Charles Perkins Centre, University of Sydney, Sydney, NSW, Australia; ${ }^{5}$ Department of Maternal, Child and Adolescent Health, Burnet \\ Institute, Sydney, NSW, Australia; ${ }^{6}$ Department of Primary Care, Health Education England, London, UK \\ Correspondence to: Mitch Blair. Department of Primary Care and Public Health, Imperial College, London, UK. Email: m.blair@imperial.ac.uk.
}

Received: 19 November 2019; Accepted: 02 April 2020; Published: 20 January 2021.

doi: 10.21037/mhealth-19-233

View this article at: http://dx.doi.org/10.21037/mhealth-19-233

We are writing to congratulate the authors of the recently published detailed evaluation of an app (Baby Buddy) designed to support parents during pregnancy and the early years (1). Building on the existing data on the limited effectiveness of mobile health applications in pregnancy and the postnatal period $(2,3)$, this paper is a valuable addition to the current literature in the field. We note that no differences were found in the primary measure of parenting self-efficacy using the TOPSE tool or the secondary outcome of the Warwick Edinburgh Mental Wellbeing Scale (WEMWBS), and this was appropriately recorded in the abstract of the paper.

Interestingly, despite the issues with retention [only $114 / 296(38.5 \%)$ of those assessed at 3 months had used the app], which the authors acknowledge, there was a difference noted in the post-hoc analysis of breastfeeding rates. On adjusted analysis (for IMD decile, education, technology use, use of pregnancy apps and baseline intention to breastfeed), the researchers found a significant increase in any breastfeeding at 1 month [aOR: 3.08 (95\% CI: 1.49 to 6.35)] and in exclusive breastfeeding at 3 months $[1.79(95 \%$ CI: 1.02 to 3.16$)]$.

Secondary to the short, medium and long-term benefits that increased breastfeeding confers on individual and population health (4), we believe that an intervention that increased breastfeeding 3-fold at 1 month and exclusivity 1.8 -fold at 3 months deserves some consideration in the commentary of the results. This behavior change component of the Baby Buddy app has been considered in relation to the COM-B framework and the behavior change wheel $(\mathrm{BCW})$ and aligns well $(5,6)$. Video content related to breastfeeding focuses on developing capability through enhancing both skills and knowledge, drivers of motivation both automatic and reflective and physical and social opportunities to breastfeed. Further, the functionality of the app addresses the intervention functions of the BCW such as enablement, incentivization, education and modelling. This behavior change framework may contribute to the differences seen for this outcome compared with the primary outcome for this particular study.

We would therefore encourage future research into mobile health applications such as Baby Buddy, using such a theoretical framework, to consider their effect on breastfeeding as well as duration and exclusivity.

\section{Acknowledgments}

Funding: None.

\section{Footnote}

Provenance and Peer Review: This article was a free submission to the journal. The article has undergone external peer review.

Conflicts of Interest: All authors have completed the ICMJE uniform disclosure form (available at http://dx.doi. org/10.21037/mhealth-19-233). Dr. MB reports he acts as $\mathrm{RCPCH}$ advisor for Baby Buddy and have been involved in its development for at least 10 years. Ms. LM is conducting 
collaborative research with the charity Best Beginnings. Dr. AG reports that LM and AG have assessed the quality of the baby buddy app and also mapped its content to the Behavior Change Wheel as part of LM PhD. The other authors have no conflicts of interest to declare.

Ethical Statement: The authors are accountable for all aspects of the work in ensuring that questions related to the accuracy or integrity of any part of the work are appropriately investigated and resolved.

Open Access Statement: This is an Open Access article distributed in accordance with the Creative Commons Attribution-NonCommercial-NoDerivs 4.0 International License (CC BY-NC-ND 4.0), which permits the noncommercial replication and distribution of the article with the strict proviso that no changes or edits are made and the original work is properly cited (including links to both the formal publication through the relevant DOI and the license). See: https://creativecommons.org/licenses/by-nc-nd/4.0/.

\section{References}

1. Deave T, Ginja S, Goodenough T, et al. The Bumps and

doi: $10.21037 /$ mhealth-19-233

Cite this article as: Blair M, Downe S, Gordon A, Musgrave L, Homer C, Etherington C. Baby Buddy app evaluationeffective uplift in breast feeding despite unclear mechanism. mHealth 2021;7:16.
BaBies Longitudinal Study (BaBBLeS): a multi-site cohort study of first-time mothers to evaluate the effectiveness of the Baby Buddy app. Mhealth 2019;5:42.

2. Daly LM, Horey D, Middleton PF, et al. The effect of mobile app interventions on influencing healthy maternal behavior and improving perinatal health outcomes: systematic review. JMIR Mhealth Uhealth 2018;6:e10012.

3. Overdijkink SB, Velu AV, Rosman AN, et al. The usability and effectiveness of mobile health technology-based lifestyle and medical intervention apps supporting health care during pregnancy: systematic review. JMIR Mhealth Uhealth 2018;6:e109.

4. Cochrane Library Special Collection. Enabling breastfeeding for mothers and babies. 2017. Available online: https://www.cochranelibrary.com/collections/ doi/10.1002/14651858.SC000027/full

5. Thomson G, Crossland N. Using the behaviour change wheel to explore infant feeding peer support provision; insights from a North West UK evaluation. Int Breastfeed J 2019;14:41.

6. Musgrave L, Baum A, Perera N, et al. Applying the Behaviour Change Wheel: insights into the development of the Breastfeeding Component of the Baby Buddy App. In: Conference on Behaviour Change, 2018. 